\title{
A Parameterized Automatic Cache Generator for FPGAs
}

\author{
Peter Yiannacouras and Jonathan Rose \\ Edward S. Rogers Sr. Department of Electrical and Computer Engineering, University of Toronto \\ Toronto, Ontario, Canada M5S $3 G 4$ \\ \{yiannac@ecf.utoronto.ca,jayar@eecg.utoronto.ca\}
}

\begin{abstract}
Soft Processors, which are processors implemented in the programmable fabric on FPGAs, are finding a multitude of applications in modern systems. An important part of processor design are the caches that have been used to alleviate the degradation in performance caused by accessing slow memory. In this paper we present a cache generator which can produce caches with a variety of associativities, latencies, and dimensions. This tool allows processor system designers to effortlessly create, and investigate different caches in order to best meet the needs of their target system. The effect of these three parameters on the area and speed of the caches is also examined and we show that the designs can meet a wide range of specifications and are in general fast and compact.
\end{abstract}

\section{Introduction}

Memories have failed to keep up with the increase in speeds seen in microprocessors [7]. This is known as the processor-memory performance gap, which is a growing problem showing no signs of abating. The most common way to bridge this performance gap is through the use of a cache, a small high speed memory which aims to satisfy memory operations alone. Caches were commercially introduced more than 30 years ago in the IBM 360/86 and have been an active area of research for even longer [4]. Since then, caches have become a salient element in today's memory hierarchy, and are consistently used in both general purpose and embedded computer systems, as well as in hard disks, web servers, and many other devices beset by slower storage components.

Soft processors, such as Altera's Nios [3] and Xilinx Microblaze [11] have recently become widely used in all manner of embedded processing systems [6]. As designers seek higher performance from these processors, caches will also become an important part of the soft processor memory hierarchy.

Choosing the best cache for a system is very application-specific, but is often done subjectively. Designers seek to minimize the silicon area consumed by a cache, and maximize its speed while achieving an effective hit ratio. Each of these issues must be dealt with by carefully selecting the many characteristics of the cache. The wide range of characteristics allow for a large range of possible cache candidates to evaluate. A particular cache is often selected based on general notions of its area, speed and effectiveness inferred from the many studies performed on caches [4]. This often leads to a less than optimal cache solution. In order to more aggressively pursue good choices for cache parameters, a more precise understanding of a cache's area, speed, and effectiveness is required. Our goal is to facilitate such a pursuit by providing an automatic cache generator for soft processors which, given a set of input parameters, will output an efficient cache implementation satisfying the given parameters. The generator is versatile as it allows for a number of different cache types to be generated. Users can select the appropriate size, latency, and associativity for their desired cache and use the proposed generator to create an implementation. While The implementations are targeted for Altera's Stratix family of FPGAs [1], the code could be modified to emit primitives specific to other families of FPGAs.

The generator can be used to easily implement a desired cache, quickly evaluate performance for a number of caches, or to compile a catalogue of statistics for several cache variants. We will present statistics for the area and speed of caches with different associativities, latencies, and dimensions in an FPGA environment. Doing so will perhaps pave the way for a more exact and deterministic process for selecting cache parameters.

The remainder of this paper is organized as follows: Section 2 describes the Stratix FPGA architecture and necessary Cache nomenclature; Section 3 describes the various cache designs that can be generated; Section 4 examines the resource utilization and speed performance for caches of various types and sizes; Section 5 provides a link to the software developed; and Section 6 concludes.

\section{Background}

\subsection{Stratix}

The Stratix FPGA from Altera is built in a 0.13um technology, and contains a heterogenous mixture of programmable logic, memory, and arithmetic blocks [1][10]. Its memory blocks come in three different sizes: 
512 bits, 4 kilobits, and 512 kilobits (not including parity bits). These memory blocks are named M512, M4K, and MRAM (Mega RAM) according to their size and will be referred to as such throughout this document. All memories are dual port memories with customizable aspect ratios. However, the M512 is confined to using one port for reading, and the other for writing. The Stratix Logic Element (LE) consists of a 4-input lookup table and flip-flop, with the ability to implement 1 bit of add/subtract arithmetic in each cell.

\subsection{Speed}

The speed of a system is defined as its maximum operational clock frequency. FPGA implementation tools report this frequency as well as the particular path within the system which can not operate faster than this reported frequency. This path is known as the critical path.

\subsection{CAD flow}

Our cache generator outputs a Verilog description of the cache and associated design files. We submit these files to Altera's Quartus CAD software package, which performs all levels of synthesis from HDL elaboration, logic synthesis, technology mapping, packing, placement, routing and programming file generation. All results reported here use Quartus II version 2.1.

\subsection{Caches}

A cache is a memory that stores a subset of the data available in a processor's address space. If the currently addressed data is found in the cache, a hit is said to have occurred and the cache can satisfy the memory operation without involving the slower memory. If the data is not found in the cache, then a miss is said to have occurred and the slower memory must be accessed. An effective cache is one which minimizes misses, hence having a high hit rate.

Data values in the cache are identified using a tag. A tag is the subsection of the address required to uniquely identify the data (see Figure 1). Tags are each stored in a tag store alongside the corresponding data stored in a data store. To detect a hit, the cache must compare the tag of the currently addressed data, to all of the relevant tags in the tag store. There are several attributes in a cache which may radically change its cost and performance, which are described below.

\subsubsection{Cache Dimensions}

There are three dimensions which define the size of a cache. These are its cache line, cache depth, and tag width. A cache line refers to the unit of data storage, in bits, used in the cache. The maximum number of cache lines that can be stored in the cache is known as the cache depth. The tag width is the number of bits in a tag as defined previously.

\subsubsection{Associativity}

Ideally, new data can be added to the cache as long as the cache has an unoccupied cache line available. This implies that a given data can map to any cache line. Such a cache is known as a fully associative cache, or just associative cache. To find a match it must search through all entries in the cache and compare it to the tag given. Because of this, a fully associative cache can be large, expensive, and slow. To alleviate these problems more simplified caches such as the direct-mapped cache have been introduced. A direct-mapped cache maps data to only one cache line determined by the low order bits of its address; these bits are referred to as the index bits. With this mapping, the cache line being read from or written to is known immediately from the address. As a result, the circuit is much simpler and faster. Between these two extremes is the set associative cache. Instead of using the index bits to select a single cache line, they are used to select a group of cache lines where the data can be placed. These selected cache lines define a set. An $n$-way set associative cache contains $n$ cache lines in each set. Since this cache needs only to search through the $n$ cache lines instead of the entire cache, it is also much simpler than the fully associative approach. Set associative caches generally provide the best compromise between circuit complexity and performance.

Note that direct-mapped and fully associative caches can be thought of as $n$-way set associative caches with $n$ equal to 1 and the cache depth respectively. As $n$ increases the number of bits required to specify the set index decreases and the tag increases (see Figure 1).

\begin{tabular}{|c|c|c|}
\hline $\mathrm{Tag}$ & Set Index & Word \\
\hline & $\log _{2}\left(\right.$ depth)- $\log _{2} n$ & \\
\hline
\end{tabular}

Figure 1. Subsections of an Address for an n-way Cache

\subsubsection{Replacement Policy}

When new data is to be cached and all available cache lines are occupied, old data must be evicted from the cache to make space. The strategy used to choose which data to evict is known as the replacement policy. The most common method is known as $L R U$ (Least Recently Used). This strategy tracks how recently each piece of data was referenced, and selects the one which was used least recently to evict. 


\subsubsection{Write Policy}

The manner with which a cache manages write operations is referred to as the cache's write policy. A write operation can potentially cause the cache and memory to become unsynchronized if the cache contains a more recent value than the memory, and that value is evicted without being written to memory. To prevent this problem, a cache can ensure that new values are flushed to memory before being evicted. A cache which behaves in this manner uses a write-back policy. Another means of dealing with this problem is to ensure the synchronization of the cache and memory by always writing to both. This is known as a write-through policy and is much simpler than the write-back policy.

\subsection{Content Addressable Memories for Fully Associative Caches}

As discussed above, a fully associative cache must search through a number of tags to find a match; this process can be done using a CAM (Content Addressable Memory). A CAM is the inverse of RAM. While a RAM is given an address and outputs the data stored at that address, a CAM receives data, often called a pattern, and returns the address where it is stored, or indicates that the pattern is not currently in the CAM. This makes CAMs ideal for searching through tags and detecting cache hits. In a cache, the pattern given to the CAM is the tag and the address returned by the CAM is the position of the associated data in the data store. The CAM used in this design was derived from an implementation described in [5]. This implementation is summarized below.

\subsubsection{Building CAMs using Block RAMs}

This CAM implementation of [5] uses a technique which manipulates RAM to be used as a CAM. The CAM pattern is used as the address to the RAM, and the data in the RAM stores the positions in the CAM where the pattern can be found. To store the 8-bit pattern with decimal value 6 in a CAM at address 2 , its representation in the RAM would be as shown in Figure 2 for a CAM with 16 words and an 8 bit input pattern.

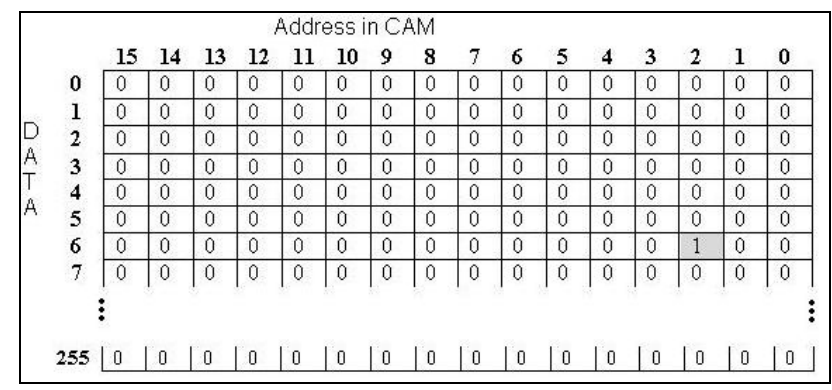

Figure 2. Representation of a CAM in RAM
In the RAM, there is a word for every possible input pattern (as seen along the left most column of Figure 1), and each entry has one bit for every possible location in the CAM. Thus an $\mathbf{m} \times \mathbf{n}$ CAM requires a $2^{\mathrm{n}} \times \mathrm{m}$ RAM. The implementation in [5] uses RAM blocks with 4096 bits which can be configured as a 256-word $x$ 16-bit RAM. This can be thus used as a 16-word x 8-bit CAM in the manner shown in Figure 2.

Using this basic approach requires a vast amount of RAM to implement even a small CAM: the amount of RAM required for an $\mathbf{m} \times \mathbf{n}$ CAM grows exponentially with the data width as seen in equation 1 , where $\mathbf{m}$ is the CAM depth and $\mathbf{n}$ the width.

$$
\# \text { bits }=m \times 2^{n}
$$

This exponential growth can be elminated, as pointed out in [5] by cascading small CAM blocks implemented with this approach, to build larger ones. For example, a 32-word deep CAM can be built out of two 16 word CAMs, or a 16-bit wide CAM can be built out of two 8bit wide CAMs. To build a deeper CAM, observe that the output of the CAM block has one bit for each word.. Thus, to add more depth, one need only increase the number of bits in the output. This can be done with some additional logic by simply using multiple CAM blocks in parallel. The additional logic is required to select the correct CAM block when write operations occur. The width of the CAM can also be extended by simply performing a logical AND of the output of multiple CAM blocks. Each CAM block receives a subsection of the input pattern; the full pattern is in the CAM if each CAM block experiences a match in the same address.

The major limitation in this design is that there is poor support for clearing locations in the CAM. Erasing the pattern in a given location would require writing a zero to the correct column of each of the 256 rows in the RAM. Using 256 cycles to erase data is impractical, hence requiring designers to address this erasing problem. We can leverage the fact that each tag appears only once in the CAM to make erasing an entry easier, as discussed in more detail in Section 3.1

In general, using a set of small M x N CAM blocks, an m $\mathrm{x}$ n CAM will require the following number of memory bits.

$$
\# \text { bits }=\frac{1}{N} 2^{N} \times m \times n
$$

As seen in equation 2, the growth is no longer exponential with $\mathrm{n}$, instead, it is exponential with the parameter $\mathrm{N}$ which is fixed and determines the CAM block width. The expression is also independent of $\mathrm{M}$, meaning CAM 
blocks can be of any depth without affecting the amount of memory used overall. As a result, it remains only to choose a suitable $\mathrm{N}$. The function $2^{N} / N$ is monotonically increasing for integer values of $\mathrm{N}$ greater than zero, hence, the optimal $\mathrm{N}$ is the minimal $\mathrm{N}$ allowed. Because of constraints on the aspect ratio of the RAM blocks in Xilinx's Virtex, the smallest possible N, or shallowest memory, occurs for $\mathrm{N}=8$. The CAM block depth $\mathrm{M}$ is then assigned a value such that it utilizes the entire 4096 bit RAM block, and hence the implementation in [5] used a 16 x 8 CAM block to build larger CAMs.

\section{Cache Design}

Table 1 lists the set of characteristics that can be specified to our parameterized cache generator.

Table 1. Characteristics of Each Cache Type

\begin{tabular}{|l|c|c|c|}
\hline & $\begin{array}{c}\text { Fully } \\
\text { Associative }\end{array}$ & $\begin{array}{c}\text { Direct- } \\
\text { mapped }\end{array}$ & $\begin{array}{c}\text { Two-way } \\
\text { Set Assoc. }\end{array}$ \\
\hline Read Latency & 2,3 & 1,2 & 1,2 \\
Write Latency & 1 & 1 & 1,2 \\
Replacement & Counter & - & LRU \\
Write Policy & Write-Thru & Write-Thru & Write-Thru \\
Depth & any & any & any \\
Addr Width & any & any & any \\
Data Width & any & any & any \\
\hline
\end{tabular}

In order to achieve faster performance and smaller area, all generated caches employ a write-through policy. For the same reason, none of the caches allow access to sub-sections of the cache line, and as a result the cache line is equal to the data width in our case and will be referred to as such throughout this document. A more detailed description of the individual cache designs and their various configurations follow.

\subsection{Fully Associative Cache}

The design of a fully associative cache involves five components: A CAM, an encoder, a data store, a tag store, and a counter. Figure 3 illustrates a read operation.

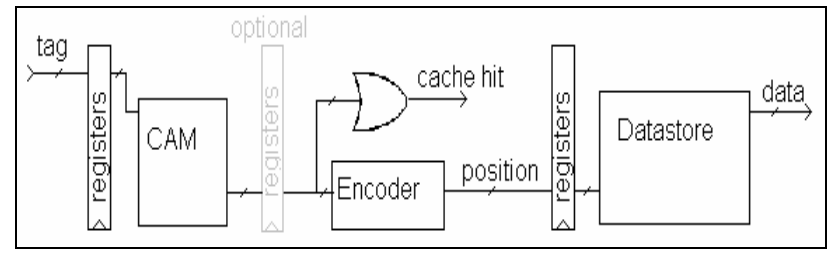

Figure 3. Associative Cache Read

A simple logical OR of the decoded CAM outputs is used to detect a hit. An encoder is used to generate the encoded address needed by the data store. The encoder is the largest block of logic in the cache and is often within the system's critical path. Its size is determined solely by the depth of the cache but is minimized in this design by capitalizing on the CAM's one-hot encoded outputs.

When a cache miss occurs the data is fetched from the memory and is passed both to the processor and used to perform a write operation into the cache. A write requires two clock cycles though the first is done in the same clock cycle the write is requested. This first cycle deletes the cached value located at the position targeted by the counter replacement algorithm. Here we have to deal with the issue of erasing a CAM entry discussed above in Section 2. To this end, a tag store is included with the associative cache, despite the fact that ideally the CAM should serve as the tag store. When data is being evicted from a given position in the cache, the tag store is used to identify its tag so that it may be erased from the CAM in a single cycle (by knowing which row has a one and assuming the other rows are zero, only one write is required). This lookup requires one clock cycle; however we can ensure that this is done before the end of any previous operation. Hence the tag to be evicted is available at the start of any subsequent operation, enabling the first cycle to be used to erase this tag, and the following cycle used to write the data to the data store and the new tag into the CAM and tag store.

A counter-based replacement policy allows for a simple way to select data to evict from the cache. The counter points at the next value to be evicted in the cache and increments with each new value cached. This method was also used in the sample code for an associative cache using Altera APEX CAMs [2]. A slight modification of it was used to save a clock cycle when incremented. This modification is shown in Figure 4.

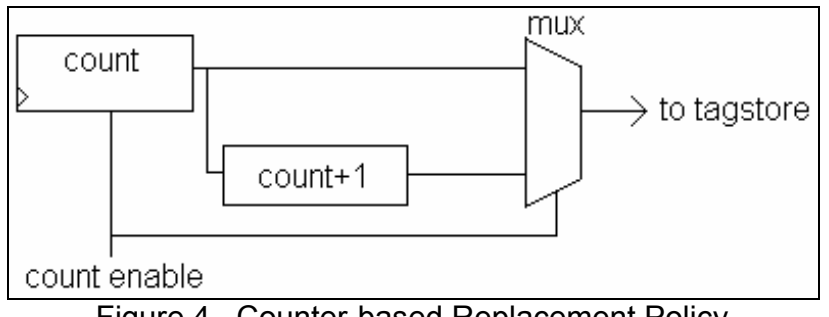

Figure 4. Counter-based Replacement Policy

The CAM implementation used in this design is identical to that in [5]; the only modification is in the chosen dimensions of the CAM blocks. The design in [5] used 16 x 8 CAM blocks implemented on 4096-bit RAM blocks on Xilinx's Virtex FPGAs. From equation (3) it can be seen that the coefficient $2^{N} / N$ is solely responsible for the inflation of memory bits required. This coefficient will be referred to as the waste factor. For $\mathrm{N}=8$, as used in [5], the waste factor is 32. With Stratix FPGA's small memory blocks, the parameter $\mathrm{N}$ can be made as small as 5 by using an M512 as a $16 \times 5$ CAM. This results in a 
waste factor of 6.4 and so $16 \times 5$ CAM blocks were used in this design.

The optional registers allows for the choice in 2 or 3 cycle read latencies. They were implemented using the built-in flip flops of the memory blocks.

\subsection{Direct-Mapped Cache}

The direct-mapped cache has a simple design. It requires only a tag store, data store, and comparator, where the tag and data stores are both single-port RAM blocks. Since only one tag in the tag store can match the tag input, that tag is the only one read, and the only one that need be compared. Thus a single comparator is used to detect a hit. A schematic of the read circuitry is shown in Figure 5.

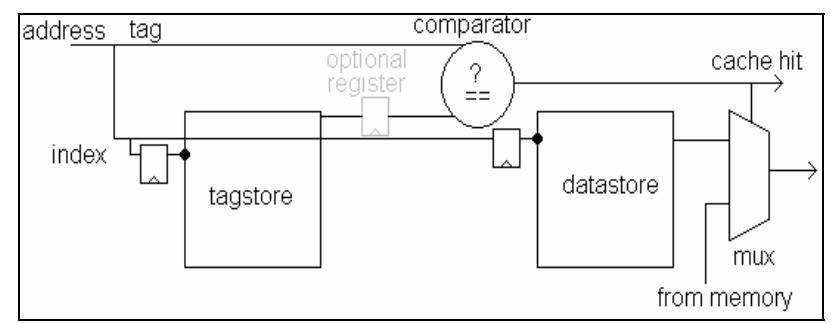

Figure 5. Cache Read for the Direct-Mapped cache

A write operation involves simply writing the tag to the tagstore and the data to the data store. This is done using the index bits as the address. No deletions are required here since the old tag will simply be overwritten.

\subsection{Two-Way Set Associative Cache}

A two-way set associative cache has two cache lines in each set. Reading from the cache requires comparing tags from both cache lines and selecting which (if any) of the data to return. Figure 6 shows the implementation of such a system which is, in effect, two direct-mapped caches arranged in parallel.

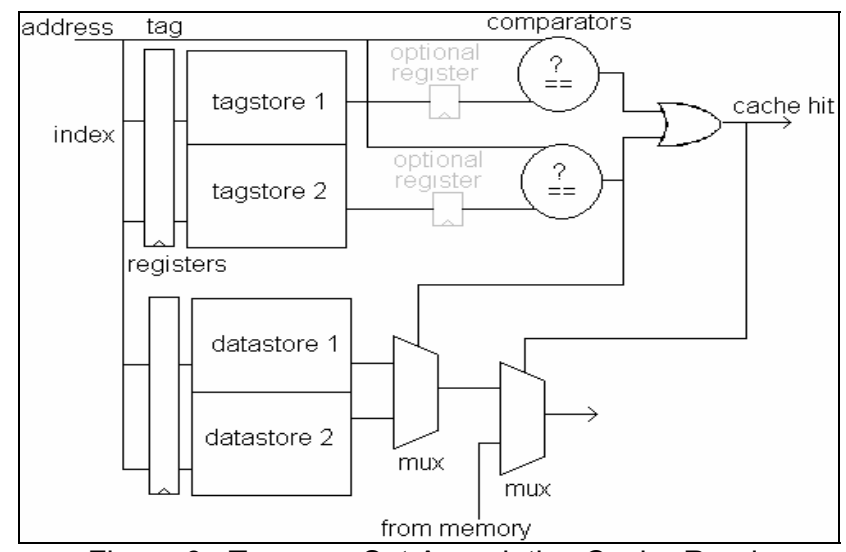

Figure 6. Two-way Set Associative Cache Read
The write operation is also identical to the directmapped cache except that a true LRU replacement policy is used to specify which of the two cache lines in a set are to be overwritten. The LRU circuitry is comprised of a single one bit-wide RAM block which indicates which of the two cache lines is to be evicted from a given set.

\section{Speed and Area Measurements}

Recall that our goal is to provide both a parameterized cache generator for soft processors, and to understand the area and speed trade-offs for the different types of caches that can be generated. In this section we compare the different types of caches with respect to their maximum operating frequency, and area in terms logic and memory usage. Factors such as latency, dimensions, and cache associativity were treated as independent variables. The dimensions of each cache is broken up into its three primary components: cache depth, memory address width, and data width. As the reader can appreciate, the complete space of possible caches to explore here is huge, and so we must limit the data collected somehow. In the foregoing, the base cache (whose parameters will be used by default) is a 32-word cache with a 32-bit address space and 32-bit data width. If any parameter is unspecified below, we will be using one of these values.

\subsection{Area}

We measure both logic (in terms of number of Stratix Logic Elements, or LEs) and memory (M512s, M4Ks, and Mega RAMs) consumption for the different caches. The effect of increased cache depth, address width, and data width will be examined for all cache types. Note that changes in latency have a negligible impact on the area of the circuits because the optional registers are already part of the Stratix memory blocks and only small changes to the control logic were needed. Thus, the measurements will be made only for the different amounts of associativity. This results are given in Figures 7 through 9.

Figure 7 shows that the number of LEs used for both the direct-mapped and two-way set associative cache is independent of the cache's depth. This is expected since the logic components of those designs include only the comparator, which depends on address width, and the output multiplexers, which depend only on the data width.

The effect of cache depth is particularly important, since deeper caches generally mean larger hit rates. The address width and data width are likely to be defined by the system but the cache depth can be independently adjusted to attain a desired hit rate. The results suggest that in the direct-mapped and set associative caches, this 
can be done without increasing the number of logic elements. Moreover, the number of logic elements is seen to be very small, respectively using $0.93 \%$ and $1.47 \%$ of the smallest Stratix chip, the S10.

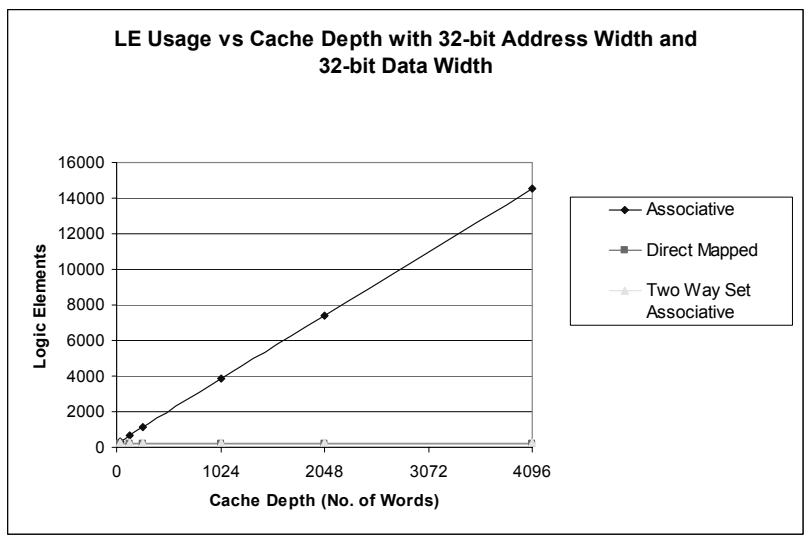

Figure 7. Graph of LE usage vs. Cache depth

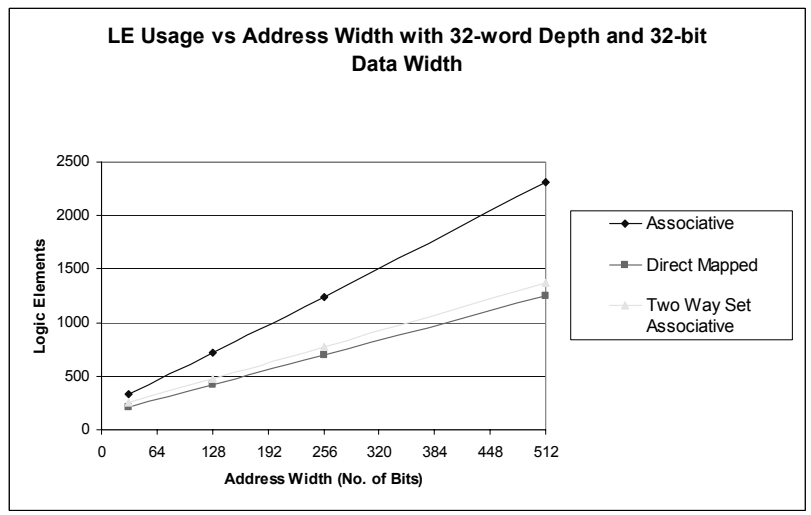

Figure 8. Graph of LE usage vs. Address Width

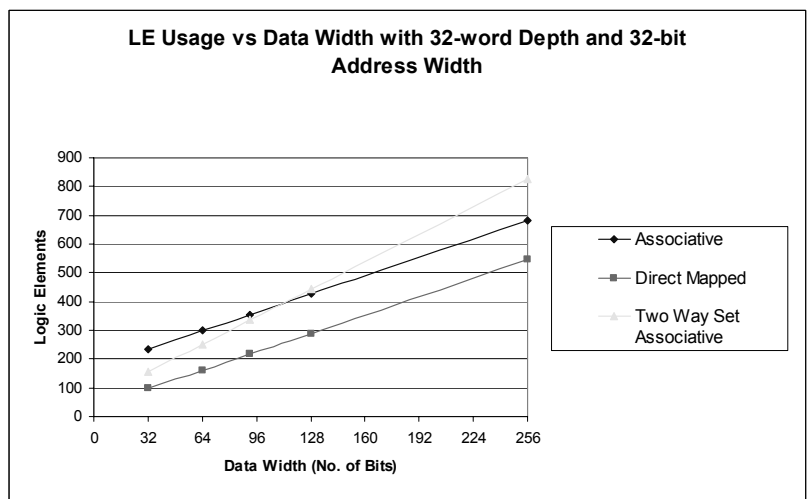

Figure 9. Graph of LE usage vs. Data Width

Conversely, the fully associative cache requires significant amounts of logic as a function of depth. The change in LEs with cache depth is linear with a slope of $3.53 \mathrm{LEs} /$ word. The contributing factors are the CAM, the encoder, the LRU circuitry, and the wide logical OR.
A 16KB (4096 words x 4 bytes/word) associative cache, which is twice the size of the Pentium 4's L1 [9] data cache, can be implemented with only 14,438 LEs.

Figure 8 shows that all cache types suffer increases in area with increases in address widths. The direct-mapped and two-way set associative cache designs both exhibit increases because of the comparators which need to compare larger tags. The two-way set associative cache increases at twice the rate of the direct mapped since it contains two comparators $(1.33 \mathrm{LEs} / \mathrm{bit}$ as opposed to $0.667 \mathrm{LEs} / \mathrm{bit}$ ). The slope values can be accounted for by considering the 2 -input function computed with 3-input luts (using carry chains). The associative cache increases at a much higher rate of $3 \mathrm{LEs} / \mathrm{bit}$. This is composed of the $1 \mathrm{LE} /$ bit for the tag mux which drives into the CAM, added to the $2 \mathrm{LEs} / \mathrm{bit}$ for cascading CAM blocks. Ideally only $1 \mathrm{LE} /$ bit is required for cascading CAM blocks, but two CAM blocks are required to expand the CAM's depth to the reference value of 32 . The LE usage is reasonably small allowing for an absurdly large 512-bit address space to use only 1673 LEs for a fully associative cache.

The effect of increasing data width size on the number of LEs used is shown in Figure 9. The only logic which depends on the data width are the two multiplexers on the data input and data output ports of the data store. Thus the growth is linear with slopes of $2 \mathrm{LEs} / \mathrm{bit}(1 \mathrm{LE} / \mathrm{bit}$ for each mux) for the associative cache and direct-mapped cache. The two-way set associative cache contains an additional data multiplexer to choose between the two lines in a give set. Thus its slope is 3 LEs/bit. Again these increases are rather moderate.

While all the designs had relatively small LE requirements, the opposite is seen in their memory requirements. The designs are quite memory intensive and this defines the upper bound on the size of the cache that can be implemented on a given FPGA. The number of Stratix M512s, M4Ks, and Mega RAMs required for each cache design is shown in Tables 2, 3 and 4. Again the latencies are ignored since this did not impact the number of memories used. Note that the Altera software system, Quartus II, selects the target physical memories used from among the 3 choices, given a logical specification. (Except in the case where we explicitly told it to use the small memories for the CAMs).

Table 2. Memories used for different Cache Depths

\begin{tabular}{|c|ccc|ccc|ccc|}
\hline & \multicolumn{8}{|c|}{ Number of RAM Blocks Used } \\
\hline \multirow{2}{*}{$\begin{array}{c}\text { Depth } \\
\text { (words) }\end{array}$} & \multicolumn{3}{|c|}{ Associative } & \multicolumn{3}{c|}{ Direct Mapped } & \multicolumn{3}{c|}{ Two Way Set Ass. } \\
\cline { 2 - 10 } & 512 & $4 \mathrm{~K}$ & Mega & 512 & $4 \mathrm{~K}$ & Mega & 512 & $4 \mathrm{~K}$ & Mega \\
\hline 32 & 14 & 2 & 0 & 0 & 2 & 0 & 1 & 4 & 0 \\
128 & 56 & 2 & 0 & 0 & 2 & 0 & 1 & 4 & 0 \\
256 & 112 & 4 & 0 & 0 & 4 & 0 & 1 & 4 & 0 \\
1024 & 448 & 16 & 0 & 0 & 14 & 0 & 1 & 14 & 0 \\
2048 & NA & NA & NA & 0 & 27 & 0 & 0 & 29 & 0 \\
4096 & NA & NA & NA & 0 & 0 & 1 & 0 & 55 & 0 \\
\hline
\end{tabular}


Table 3. Memories used for different Address Widths

\begin{tabular}{|c|ccc|ccc|ccc|}
\hline \multirow{2}{*}{$\begin{array}{c}\text { Addr. } \\
\text { Width }\end{array}$} & \multicolumn{10}{|c|}{ Number of RAM Blocks Used } \\
\cline { 2 - 10 } (bits) & Associative & & \multicolumn{1}{|c|}{ Direct Mapped } & \multicolumn{2}{|c|}{ Two Way Set Ass. } \\
\cline { 2 - 10 } & 512 & $4 \mathrm{~K}$ & Mega & 512 & $4 \mathrm{~K}$ & Mega & 512 & $4 \mathrm{~K}$ & Mega \\
\hline 32 & 14 & 2 & 0 & 0 & 2 & 0 & 1 & 4 & 0 \\
128 & 53 & 4 & 0 & 1 & 4 & 0 & 3 & 8 & 0 \\
256 & 105 & 8 & 0 & 0 & 8 & 0 & 3 & 16 & 0 \\
512 & 207 & 15 & 0 & 0 & 15 & 0 & 3 & 30 & 0 \\
\hline
\end{tabular}

Table 4. Memories used for different Data Width

\begin{tabular}{|c|ccc|ccc|ccc|}
\hline \multirow{2}{*}{$\begin{array}{c}\text { Data } \\
\text { Width }\end{array}$} & \multicolumn{8}{|c|}{ Number of RAM Blocks Used } \\
\cline { 2 - 10 } (bits) & \multicolumn{1}{|c|}{ Associative } & & \multicolumn{3}{|c|}{ Direct Mapped } & \multicolumn{3}{c|}{ Two Way Set Ass. } \\
\cline { 2 - 10 } & 512 & $4 \mathrm{~K}$ & Mega & 512 & $4 \mathrm{~K}$ & Mega & 512 & $4 \mathrm{~K}$ & Mega \\
\hline 32 & 14 & 2 & 0 & 0 & 2 & 0 & 1 & 4 & 0 \\
64 & 14 & 3 & 0 & 0 & 3 & 0 & 1 & 6 & 0 \\
92 & 14 & 4 & 0 & 1 & 3 & 0 & 1 & 8 & 0 \\
128 & 14 & 5 & 0 & 1 & 4 & 0 & 1 & 10 & 0 \\
256 & 15 & 8 & 0 & 0 & 8 & 0 & 1 & 17 & 0 \\
\hline
\end{tabular}

As suggested in equation (2), the growth of the M512s used for the CAMs is linear. Changes in the data width obviously have no effect on the CAM and this too can be seen in Table 4.

An interesting observation occurs for the directmapped cache with depth of 4096 words. The Quartus II compiler merged the tag store and data store into a single Mega RAM block, since they have common address lines. Implementing the design in this manner has two main advantages. The first advantage is that it recovers memory blocks. Only 1 Mega RAM block and 98 LEs are needed to implement the $16 \mathrm{~KB}$ direct-mapped cache. This enables the design to fit on the smallest Stratix, the S10, while using less than $1 \%$ of its logic elements and none of its M512 or M4K memories. The second advantage is that it simplifies routing which may speed up the circuit. The disadvantage is that the Mega RAMs are reported as being up to $35 \mathrm{MHz}$ slower than the M4K [1]. We discuss the speed the cache designs following more detail below.

\subsection{Speed}

The essential feature of caches are their speed, which has to be significantly faster than the main store. In this section we present the effect of different cache parameters and types on the post-routed speed of the cache. Recall that critical path is measurement by the Quartus II timing analyzer. Data was gathered for all cache types including different latencies since latency plays a pivotal role in the system's overall speed. Figures 11, 12 and 13 provide graphs generated from the measured data.

As expected the direct-mapped cache is generally fastest and the associative cache is slowest. It is interesting to note that an associative cache with a three cycle read latency can outperform a single cycle read latency direct-mapped cache. This shows that read latency plays a major role in determining maximum operational frequency. On the other hand, the write latency has little effect since the write circuitry is not part of the critical path except in very small caches. A minute cache with dimensions $16 \times 10 \times 8$, produced speed ups of up to $70 \mathrm{MHz}$ by increasing write latency.

Increases in address width resulted in signficant speed reductions in all caches while increases in data width had little effect. Increases in cache depth is seen to adversely affect only the associative cache. These results follow intuitively from growth in their respective components as it is mostly larger CAMs and comparators that slow down the larger caches. Addresses affect both, data widths change neither, and depth influences only CAMs.

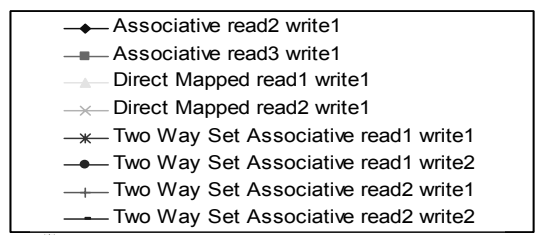

Figure 10. Legend for Figures 10-12

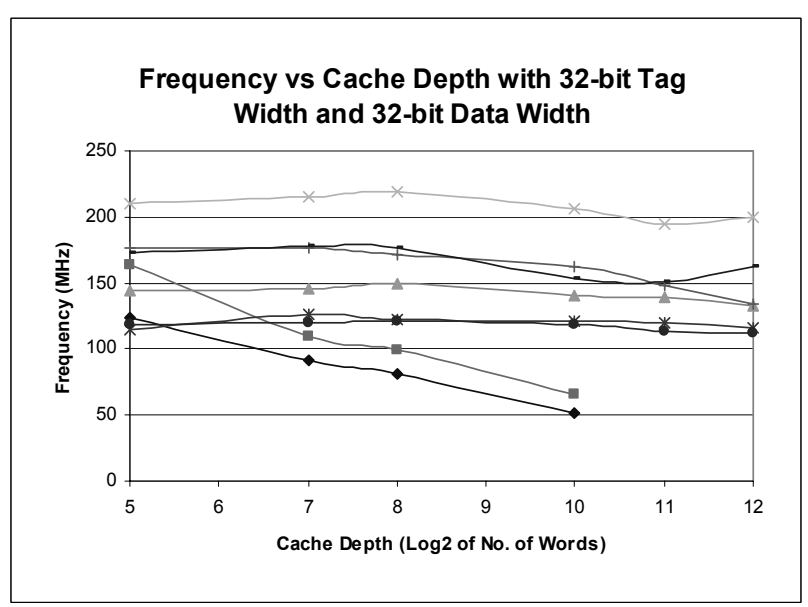

Figure 11. Graph of Frequency vs. Cache Depth 


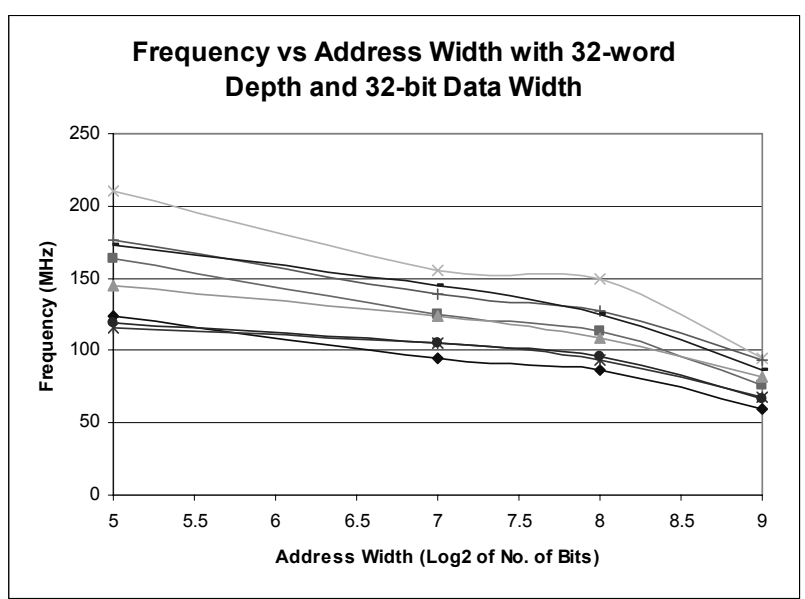

Figure 12. Graph of Frequency vs. Address Width

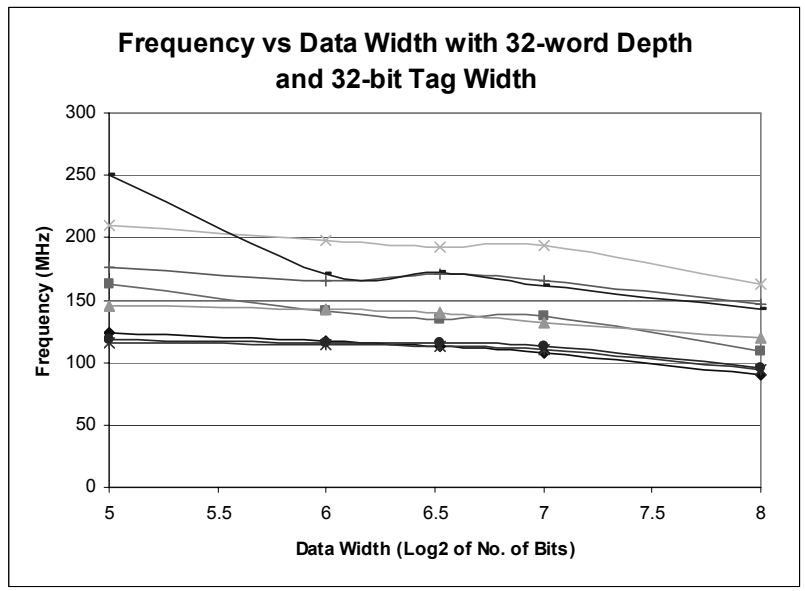

Figure 13. Graph of Frequency vs. Data Width

\section{Software}

The software and source code for the cache generator can be found at the following URL: http://www.eecg.toronto.edu/ jayar/software/cachegen/ca chegen.html

\section{Conclusion}

We have presented an automatic parameterized cache generator that emits cache designs for an FPGA in a Verilog output file. The input to the generator is a set of parameters describing the desired cache, including associativity, latency, cache depth, address width, and data width. Cache designs were generated and evaluated for a wide variety of input parameters. From this analysis, a number of trends were established concerning their area and speed. The generator is very robust offering a wide variety of cache types, able to satisfy a wide variety of size and speed constraints. With this tool, and the presented statistics, a designer can identify cache parameters which satisfy the area and speed constraints of a system, and then choose the cache with optimal effectiveness. Progress in this direction will eventually yield a more deterministic method of selecting cache types for soft processors.

\section{References}

[1] Altera Corporation. "Altera Stratix FPGA Family Data Sheet," December 2002.

http://www.altera.com/literature/ds/ds_stx.pdf

[2] Altera Corporation, "AN 119: Implementing High-Speed Search Applications with Altera CAM," in Altera Application Notes, July 2001.

http://www.altera.com/literature/an/an119.pdf

[3] Altera Corporation, "Nios Soft Core Embedded Processor Data Sheet," July 2003.

http://www.altera.com/literature/ds/ds_excnios.pdf

[4] J.L. Baer, Department of Computer Science \& Engineering, University of Washington. "2K papers on caches by Y2K: Do we need more?". November 2000, http://www.irit.fr/ACTIVITES/EQ_APARA/HPCA6/Baer Hpca6.PDF

[5] J.L. Brelet. "Using Block RAM for High Performance Read/Write CAMs" in Xilinx Application Note xapp204. May 2000, http://www.xilinx.com/xapp/xapp204.pdf

[6] A. Cataldo, "Altera, Xilinx heat up processor-core fray", EE Times, October 2001, http://www.eetimes.com/story/OEG20011016S0097

[7] J. L. Hennessy and D. A. Patterson. "Computer Architecture: A Quantitative Approach, $3^{\text {rd }}$ edition". Morgan Kaufmann Publishers, 2003.

[8] C. Hamacher, Z. Vranesic, and S. Zaky. "Computer Organization", $5^{\text {th }}$ edition. McGraw-Hill, 2002.

[9] Intel Corporation. "Intel ${ }^{\circledR}$ Pentium ${ }^{\circledR} 4$ Processor with 512KB L2 Cache on 0.13 Micron Process at $2 \mathrm{GHz}-3.06$ $\mathrm{GHz}$, with Support for Hyper-Threading Technology 1 at 3.06 GHz Datasheet," January 2003.

ftp://download.intel.com/design/Pentium4/datashts/298643 07.pdf

[10] D. Lewis, V. Betz, D. Jefferson, A. Lee, C. Lane, P. Leventis, S. Marquardt, C. McClintock, B. Pedersen, G. Powell, S. Reddy, C. Wysocki, R. Cliff, and J. Rose, "The Stratix Routing and Logic Architecture" in FPGA '03, ACM. Symp. FPGAs, February 2003, pp. 15-20.

[11]Xilinx Inc., "Microblaze Processor Reference Guide", April, 2003.

http://www.xilinx.com/ise/embedded/mb_ref_guide.pdf 